\title{
Highlights from the European Society of Cataract and Refractive Surgeons Annual Meeting
}

\author{
An Expert Interview with Farhad Hafezi
}

ELZA Institute, Zurich, Switzerland

DOI: https://doi.org/10.17925/EOR.2019.13.1.15

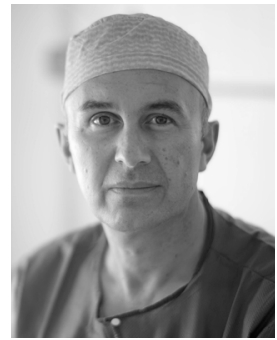

\section{Farhad Hafezi}

Farhad Hafezi, MD, PhD, is a professor of ophthalmology, a Swiss eye surgeon and researcher. He is Medical Director of the ELZA Institute, Zurich, Switzerland; Clinical Professor of Ophthalmology at the Keck School of Medicine, University of Southern California, LOS Angeles, USA; Research Group Leader at the Center for Applied Biotechnology and Molecular Medicine in the University of Zurich: Professor of Ophthalmology at the University of Geneva, Switzerland; and Visiting Professor at Medical University Wenzhou, China. Prof. Hafezi is a pioneer of corneal cross-linking (CXL) for treating keratoconus and translating CXL principles to new applications. His scientific work comprises more than 185 peer-reviewed original articles, letters, reviews and book chapters with over 7,500 citations, a cumulative impact factor of 625 and an $h$-index of 45

\section{Keywords}

ESCRS, corneal cross-linking, CXL, keratitis, corneal strength

Disclosure: Farhad Hafezi is the co-founder of the Light for Sight foundation, which administrates the K-MAP study, and is Chief Scientific and Medical Officer of EMAGine AG, who market a slit lamp-mountable cross-linking device.

Review Process: This is an expert interview and as such, has not undergone the journal's standard peer review process.

Compliance with Ethics: This article is an exper interview and does not report on new clinical data, or any studies with human or animal subjects performed by the author.

Authorship: The named author meets the International Committee of Medical Journal Editors (ICMJE) criteria for authorship of this manuscript, takes responsibility for the integrity of the work as a whole, and has given final approval for the version to be published.

Access: This article is freely accessible at tOUChOPHTHALMOLOGY.COM.

C) Touch Medical Media 2019.

Received: 18 December 2018

Published Online: 27 September 2019

Citation: European Ophthalmc Review.

2019;13(1):15-6

Corresponding Author: Farhad Hafezi, ELZA Institute AG, Webereistrasse 2,8953 Dietikon, Zurich, Switzerland. E: fhafezi@elza-institute.com

Support: No funding was received in

the publication of this article.
T he past decade has seen a number of important advances in the management and understanding of corneal disease. Corneal cross-linking $(\mathrm{CXL})$ is a technique that combines ultraviolet (UV) light and the chromophore, riboflavin, to stiffen and strengthen the cornea. The introduction of CXL has transformed the management of corneal ectasias such as keratoconus, post-LASIK ectasia and pellucid marginal degeneration. ${ }^{1,2}$ Recent scientific and technological advances have given researchers greater insight into how the CXL reaction works, and this has not only enabled modifications of the original "Dresden" cross-linking protocol, ${ }^{3}$ but also the development of new indications for $\mathrm{CXL}$, such as the treatment of infectious keratitis. Clinical research has also revealed a number of other factors that can affect corneal strength and shape, such as pregnancy and hypothyroidism.

In an expert interview, filmed at the European Society of Cataract and Refractive Surgeons (ESCRS) Annual Meeting, 22-26 September 2018, Prof. Farhad Hafezi describes his latest research in corneal disease.

\section{Q. What were your personal highlights of the ESCRS meeting?}

The city of Vienna, which is wonderful and so welcoming of international guests; and the Messe Wien Congress Centre, which is one of the best and most well-organised venues for large international congresses like the ESCRS Annual Meeting. The increased interest in corneal biomechanics from both 'pure' clinicians as well as industry. It's becoming clear that a better understanding of corneal biomechanics means not just better screening, but also better safety, quality and predictability of the patient's outcomes. If we're going to push refractive surgery further forward (both in terms of therapeutic and elective surgery on the cornea), we need to know more about how the cornea is built; how it responds to interventions like laser ablation and UV-riboflavin cross-linking; and what happens in the longer term in terms of its strength, structure and shape. I'm glad this is being recognised by so many.

I am also glad that clinicians with relatively little research experience have expressed a desire to engage in research projects like the K-MAP study (ClinicalTrials.gov Identifier: NCT03115710; that examines the global prevalence of keratoconus). K-MAP has a simple objective and a clear protocol, and this has given clinicians, who are new to research, a chance to contribute to the scientific community as well as help the study group achieve a large collection of data to achieve statistical significance. We believe that the study will solidify the belief that keratoconus should not be considered a rare disease, but in fact a far more common one. 


\section{Q. How may reducing the corneal temperature improve the efficacy of cross-linking procedure and what are the optimum means of achieving this?}

Our research group and collaborators have shown the importance of oxygen availability for the UV-riboflavin cross-linking reaction to occur; ${ }^{3,4}$ this is why we are seeing numerous attempts to increase the amount of oxygen available in the cornea, with oxygenating goggles and such like. But when we were looking at the literature, we noticed previous work showing that lowering the temperature of a tissue increases the amount of oxygen that diffuses into it. ${ }^{5}$ Therefore, in theory, cooling the cornea should increase the availability of oxygen for the cross-linking reaction, thereby improving the efficacy of the procedure. Our preliminary, ex vivo laboratory data appear to confirm that.

If the laboratory work confirms this, the next step is to cool the patient's cornea, perhaps by instilling chilled fluids, such as balanced salt solution beforehand (as is also done with photorefractive keratectomy), instilling chilled riboflavin, or perhaps by applying something like a pre-cooled bandage contact lens for a short period before and after cross-linking is performed.

\section{Q. Could you tell us a little about your studies investigating the relationship between thyroid and corneal disease, and their findings to date?}

We started looking at the influence of hormones (namely oestrogen) on corneal topography about a decade ago, as we know oestrogen can soften collagen in the body in advance of childbirth., ${ }^{6,7}$ Assessing the relationship between oestrogen and the corneal thickness and biomechanical strength is important for two main reasons: refractive surgery candidate screening, and the detection and treatment of corneal ectasias like keratoconus (which pregnancy can reveal). In fact, the relationship between thyroid hormone levels and corneal thickness was identified thanks to a collaboration we had with the Department of Obstetrics at the University of Geneva where we examined the corneal topography of healthy pregnant women during their pregnancy and for 6 months afterwards. One of the standard blood tests during pregnancy is T3/T4 (thyroid hormone) levels - and lower levels of T3/T4 (hypothyroidism) correlated most with thinner corneas. ${ }^{8}$

Looking at the literature, the first indication of keratoconus and hypothyroidism goes back to 1936, but we've since seen epidemiological data that keratoconus levels are around 10 times greater in patients with thyroid dysfunction. If you're a doctor who has patients with hypothyroidism, I'd suggest considering referring them to an ophthalmologist for keratoconus screening, and likewise, ophthalmologists who have patients with corneal ectasia under their care, refer them for a blood test to have their T3/T4 levels checked. In both cases, the patient will benefit.

\section{Q. What advances in corneal disease do you expect to emerge in 2019 ?}

Many groups are showing that keratoconus is not rare and should not be treated as such. While the verdict is still out about the root cause of the disease, we know that there are significant modulators like eye rubbing, dry climate, genetic disposition and hormonal factors. In 2019, there will be many more cross-discipline collaborations and approaches to detecting keratoconus, starting with endocrinology.

Performing laser refractive surgery on a patient with an undetected cone (or other ectasia) is disastrous, and the key to avoiding that is more and better screening. Armed with the knowledge we've gained recently on the causes of corneal ectasias, it makes sense that more people will be screened more thoroughly, making what is an extremely safe and successful procedure even more so.

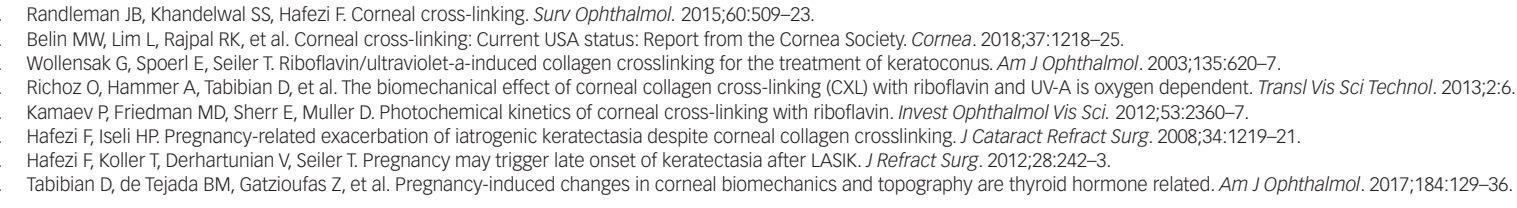

